\title{
Regional changes in type 1 cannabinoid receptor availability in Parkinson's disease in vivo
}

\author{
Koen Van Laere ${ }^{\mathrm{a}, *}$, Cindy Casteels ${ }^{\mathrm{a}}$, Sophie Lunskens ${ }^{\mathrm{b}}$, Karolien Goffin ${ }^{\mathrm{a}}$, Igor D. Grachev ${ }^{\mathrm{c}}$, \\ Guy Bormans ${ }^{\mathrm{d}}$, Wim Vandenberghe ${ }^{\mathrm{b}}$ \\ ${ }^{a}$ Department of Nuclear Medicine, University Hospitals Leuven, Leuven, Belgium \\ ${ }^{b}$ Department of Neurology, University Hospitals Leuven, Leuven, Belgium \\ ${ }^{c}$ Imaging Research Laboratories, Merck Inc, West Point, PA, USA \\ ${ }^{d}$ Laboratory of Radiopharmaceutical Chemistry, K.U. Leuven, Leuven, Belgium
}

Received 26 October 2010; received in revised form 12 January 2011; accepted 13 February 2011

\begin{abstract}
The type 1 cannabinoid receptor (CB1) is a crucial modulator of synaptic transmission in brain and has been proposed as a potential therapeutic target in Parkinson's disease (PD), especially for treatment of levodopa-induced dyskinesias (LID). Our aim was to measure CB1 levels in brains of PD patients in vivo and to investigate the relation between CB1 availability and LID. We studied 12 healthy controls and 29 PD patients (9 drug-naïve patients with early PD, 10 patients with advanced PD and LID, and 10 patients with advanced PD without LID). PD patients were examined using the Unified Parkinson's Disease Rating Scale (UPDRS) and the modified Abnormal Involuntary Movement Scale (mAIMS). All subjects underwent positron emission tomography (PET) with the CB1-selective radioligand $\left[{ }^{18} \mathrm{~F}\right] \mathrm{MK}-9470$ and magnetic resonance imaging (MRI). PD patients showed an absolute decrease in CB1 availability in the substantia nigra. By contrast, CB1 availability was relatively increased in nigrostriatal, mesolimbic, and mesocortical dopaminergic projection areas. CB1 availability did not differ significantly between advanced PD patients with and without LID. Within the group of PD patients with LID, there was no significant correlation between CB1 availability and LID severity. These data demonstrate regional changes in CB1 availability in PD in vivo, but do not support a role for dysregulation of CB1 levels in the pathogenesis of LID.
\end{abstract}

(C) 2012 Elsevier Inc. All rights reserved.

Keywords: CB1 receptor; PET; Parkinson's disease; Levodopa induced dyskinesia; Endocannabinoid system

\section{Introduction}

The motor features of Parkinson's disease (PD) result mainly from degeneration of nigrostriatal dopaminergic neurons. However, deficits in nondopaminergic transmitter systems also contribute to the parkinsonian motor symptoms and lead to various nonmotor problems such as dementia, apathy, and depression (Fox et al., 2008). One of the nondopaminergic systems that may be involved in PD pathogenesis is the endocannabinoid system (ECS) (Benar-

\footnotetext{
* Corresponding author at: Division of Nuclear Medicine, University Hospitals Leuven, Herestraat 49, 3000 Leuven, Belgium. Tel.: +32 1634 37 15; fax: +3216343759.

E-mail address: koen.vanlaere@uzleuven.be (K. Van Laere).
}

roch, 2007; Brotchie, 2003; Katona and Freund, 2008). The ECS in brain consists mainly of the type 1 cannabinoid receptor (CB1) and endogenous agonists (endocannabinoids) (Piomelli, 2003; Wilson and Nicoll, 2002). CB1 is abundantly expressed on presynaptic glutamatergic and GABAergic terminals, where its activation suppresses neurotransmitter release (Katona and Freund, 2008; Wilson and Nicoll, 2002). The ECS is a key modulator of synaptic transmission and is involved in motor behavior, cognition, and emotion (Benarroch, 2007; Brotchie, 2003; Di Marzo and Matias, 2005; Katona and Freund, 2008). The basal ganglia contain among the highest concentrations of CB1 and endocannabinoids of all brain regions (Berrendero et al., 1999; Bisogno et al., 1999; Herkenham et al., 1990).

Numerous studies have suggested a profound distur- 
bance of the ECS in PD (Benarroch, 2007; Brotchie, 2003; van der Stelt and Di Marzo, 2003). CB1 levels are increased in postmortem striatal tissue from 1-methyl-4-phenyl1,2,3,6-tetrahydropyridine (MPTP)-lesioned parkinsonian monkeys and PD patients (Lastres-Becker et al., 2001). Endocannabinoid levels are increased in cerebrospinal fluid (CSF) of untreated PD patients compared with non-PD controls (Pisani et al., 2010). An ECS-dependent form of synaptic plasticity was found to be absent in PD animal models (Kreitzer and Malenka, 2007). Particular attention has focused on the possible role of the ECS in levodopainduced dyskinesias (LID). The CB1 agonist nabilone reduced LID in MPTP-lesioned monkeys and PD patients (Fox et al., 2002; Sieradzan et al., 2001). This favorable effect on LID suggests that the ECS might be (regionally) hypoactive in PD patients with LID compared with those without LID. One possible reason for ECS hypoactivity could be downregulation of CB1. However, another clinical trial found no effect of oral cannabis extract on LID (Carroll et al., 2004).

In vivo imaging of $\mathrm{CB} 1$ in human brain has recently become feasible using quantitative positron-emission tomography (PET) with the CB1-selective radioligand $\left[{ }^{18} \mathrm{~F}\right]$ MK-9470 (Burns et al., 2007; Sanabria-Bohorquez et al., 2010; Van Laere et al., 2009, 2010). Here, we have used $\left[{ }^{18} \mathrm{~F}\right] \mathrm{MK}-9470$ PET to answer 2 questions. First, are there any regional differences in CB1 levels between PD patients and healthy controls in vivo? Second, is there a relationship between CB1 availability and LID?

\section{Methods}

\subsection{Subjects}

Twenty-nine patients with PD were recruited in the Movement Disorders Clinic of University Hospitals, Leuven. Inclusion criteria for PD patients were: diagnosis of idiopathic PD according to the United Kingdom Parkinson's Disease Society Brain Bank criteria; ability to understand the patient information brochure, and give written informed consent. Patients with previous stereotactic brain surgery were excluded. Three types of PD patients were recruited: patients with early PD, defined as drug-naïve PD patients with disease duration of $<5$ years and Hoehn-Yahr stage $<$ 2; patients with advanced PD and LID, defined as patients with disease duration of $>5$ years and clinically observable, typical levodopa-induced peak-dose dyskinesias; and patients with advanced PD without LID, defined as patients with disease duration of $>5$ years who had never experienced levodopa-induced peak-dose dyskinesias based on clinical observation and careful interviews with patients and caregivers. All advanced PD patients were levodopa-responsive and none had signs suggestive of progressive supranuclear palsy, multiple system atrophy, or corticobasal degeneration. In all early PD patients the diagnosis was confirmed with ${ }^{123}$ I-FP-CIT (ioflupane) single-photon emis- sion computed tomography (SPECT) imaging. Twenty-six of the 29 patients had sporadic PD; 3 had a family history of PD (1 had an affected father, 1 an affected mother, and 1 an affected maternal uncle). Genetic testing for mutations in the LRRK2 and PARK2 genes was performed in 2 patients, but no mutations were found. Subjects with neuropsychiatric diseases other than $\mathrm{PD}$, with major internal medical conditions, with a history of alcohol abuse, or use of marijuana or other recreational drugs were excluded.

Healthy volunteers were prospectively recruited with advertisements in the hospital, local community newspapers, and departmental homepage. In total, 50 healthy subjects between 18 and 70 years were recruited as controls for different ongoing studies. Age- and gender-matching were performed in this study, and 12 age- and gender-matched controls were retained (age range: $30.7-68.5$ years). Inclusion and exclusion criteria for healthy controls were described previously (Van Laere et al., 2008).

All PD patients were clinically evaluated on the day of PET using the complete Unified Parkinson's Disease Rating Scale (UPDRS). In the advanced group, the clinical examination was performed in the "on medication" condition to allow evaluation of LID. LID were assessed using the modified Abnormal Involuntary Movement Scale (mAIMS) during rest and serial 7 subtraction test (Guy, 1976). The levodopa equivalent daily dose (LEDD) was calculated using the following conversion rates: $100 \mathrm{mg}$ of standard levodopa was equivalent to $133 \mathrm{mg}$ of controlled-release levodopa, $75 \mathrm{mg}$ of levodopa plus entacapone, $1 \mathrm{mg}$ of pergolide or pramipexole, or $5 \mathrm{mg}$ of ropinirole. All subjects underwent physical examination, and blood and urine testing to exclude major internal pathology. Urine toxicology for cannabis and all major known addictive drugs was performed as described previously (Van Laere et al., 2008). Only nonsmokers (for at least 6 months before the PET study) were included. Handedness was evaluated with the Briggs and Nebes questionnaire (Briggs and Nebes, 1975).

\subsection{Radiotracer preparation and imaging}

The $\left[{ }^{18} \mathrm{~F}\right]$ MK-9470 precursor was obtained from Merck Research Laboratories and labeled at the PET site using ${ }^{18}$ F-ethylbromide (Burns et al., 2007). The final product was obtained after high performance liquid chromatography (HPLC) separation and had a radiochemical purity $>95 \%$. Specific activity was higher than $200 \mathrm{GBq} / \mu \mathrm{mol}$. PET acquisitions were performed using an HR + camera (Siemens, Ehrlangen, Germany). All subjects fasted for at least 4 hours before the measurements and all measurements took place between $11 \mathrm{Am}$ and 6 PM. Subjects received $310 \pm 56 \mathrm{MBq}$ of $\left[{ }^{18} \mathrm{~F}\right]-\mathrm{MK}-9470$ in slow intravenous (IV) injection. Dynamic data were acquired and images were reconstructed using a standard 3-dimensional filtered backprojection algorithm including scatter and measured attenuation correction (using a ${ }^{68} \mathrm{Ge}$ source with $2 \mathrm{D}$ acquisition). The resulting transverse and axial spatial resolution was approx- 
imately $4 \mathrm{~mm}$. For quantification only the frames between 90 and 120 minutes postinjection were retained and summed $(6 \times 300$ seconds $)$.

All subjects underwent high-resolution magnetic resonance imaging (MRI), both T1-weighted 3D-magnetization prepared rapid acquisition gradient echo (MPRAGE) and T2-weighted, on a 1.5 Tesla Vision Scanner (Siemens, Germany). Parameters for the T1 3D-MPRAGE sequence were: relaxation time $\mathrm{TR}=0 \mathrm{~ms}$, echo time $\mathrm{TE}=4 \mathrm{~ms}$, flip angle $=12^{\circ}$, inversion time $=300 \mathrm{~ms}$, matrix $256 \times$ 256, 160 contiguous sagittal slices of $1 \mathrm{~mm}$.

\subsection{Image processing}

Correction for motion between dynamic scan frames was performed using the realignment module in SPM2 (Statistical Parametric Mapping, Wellcome Department of Imaging Neuroscience, London, UK). Parametric maps of CB1 availability were constructed using modified standard uptake value (mSUV) values (Thie et al., 2007). mSUV values were determined by dividing the calibrated activity at this timeframe by the amount of tracer injected and correcting for the subject's weight: $\mathrm{mSUV}=$ activity $(\mathrm{kBq} / \mathrm{cc}) /$ injected dose $(\mathrm{MBq}) \times[($ weight $(\mathrm{kg})+70) / 2]$. This index was calculated from the area under the curve in the interval between 90 and 120 minutes postinjection and was validated previously to give reliable estimates of receptor volume of distribution as determined by full kinetic modeling (Sanabria-Bohorquez et al., 2010). Weight and injected activity were not significantly different between PD patients and controls. Possible group differences in peripheral metabolism that could lead to bias in CB1 availability determination by this index were excluded by relating $\mathrm{mSUV}$ for all healthy controls and the first 10 PD patients (5 early, 5 advanced with LID) to the corresponding fractional uptake ratio (FUR) values (Sanabria-Bohorquez et al., 2010). FUR values take into account the integral of the metabolite corrected input curve and are strongly correlated to total distribution volume $\mathrm{V}_{\mathrm{T}}$ of $\left[{ }^{18} \mathrm{~F}\right]$ MK-9470 (Sanabria-Bohorquez et al., 2010). There was no significant difference in FUR versus mSUV relationship between PD patients and controls or between PD subgroups. A direct comparison of the area under the metabolite corrected arterial input curve also did not show any significant difference.

Reconstructed data were corrected post hoc for partial volume effects using the Alfano method in the software package PVEout as published (Quarantelli et al., 2004). Segmentation of T1 MPRAGE images was performed using SPM2, while additionally the caudate, putamen, pallidum, thalamus, and hypothalamus masks were delineated manually on the transverse $\mathrm{T} 1$ slices $\left(1 \mathrm{~mm}^{3}\right.$ voxel size), after sagittal reorientation along the anterior-posterior commisural (AC-PC) line (Haznedar et al., 2006). For each subject, parametric mSUV images were spatially normalized to the standard Montreal Neurological Institute (MNI) space using a previously created $2 \times 2 \times 2 \mathrm{~mm} \mathrm{CB} 1$ template. In case of lateralized clinical affliction, image data were flipped so that "left" corresponded to the clinically most affected hemicorpus.

\subsection{Analysis design}

Data were analyzed using SPM2 on a voxel-by-voxel basis. For analysis of absolute receptor availability, no proportional scaling was used and a relative (gray matter) analysis threshold of $80 \%$ was used to exclude extracerebral, white matter, and ventricular activity. For regional analysis, proportional scaling was used. We explored group differences using $p_{\text {height }}<0.05$ corrected for multiple comparisons, and exploratory correlations at $p_{\text {height }}<0.001$ (uncorrected), with an extent threshold $k_{\text {ext }}>50$ voxels $\left(\sim 0.4 \mathrm{~cm}^{3}\right)$ In addition to an analysis of group differences, a correlation analysis with dyskinesia parameters (total score on dyskinesia items 32, 33, and 34 of UPDRS-IV, and mAIMS score) was performed in a "single subject, covariates only" design in SPM. The analyses were done with and without age, gender, disease duration, levodopa dose, and dopamine agonist dose as nuisance variables.

Conventional statistics were performed using Statistica v 8.0 (Statsoft Inc, Tulsa, OK, USA).

\section{Results}

\subsection{Patient characteristics}

We studied 12 healthy controls and 3 groups of PD patients (9 drug-naïve patients with early PD and unilateral motor signs; 10 patients with advanced PD and LID; 10 patients with advanced PD without LID). Mean disease duration in the early PD group was approximately 6 times shorter than in the advanced PD patients (Table 1). There was no significant difference in disease duration, HoehnYahr scores, dose of levodopa, dopamine agonists, entacapone, amantadine, or total LEDD between the advanced PD patients with and without LID. The mean dose of selegiline was significantly higher in the advanced group with LID than in those without LID (Table 1). Despite their similar disease duration, patients with advanced PD without LID were significantly older than those with LID, consistent with the known higher susceptibility of younger PD patients to development of LID (Fabbrini et al., 2007). Therefore, subsequent analyses were performed with and without age, gender, levodopa dose, and dose of dopamine agonists as nuisance variables, to exclude a confounding influence of these variables.

\subsection{Comparison of cerebral CB1 distribution between PD patients and controls}

PD patients showed a single significant cluster of absolute decrease of CB1 availability in a bilateral ventral mesencephalic region $\left(p_{\text {height }}<0.05\right.$ corrected for multiple comparisons) comprising the substantia nigra (Fig. 1A and B; peak cluster locations in Supplementary Table 1A). 
Table 1

Demographic and clinical characteristics of subjects

\begin{tabular}{|c|c|c|c|c|}
\hline & Controls & PD, early & PD, advanced with LID & PD, advanced without LID \\
\hline$n$ & 12 & 9 & 10 & 10 \\
\hline Gender & $6 \mathrm{~F} / 6 \mathrm{M}$ & $4 \mathrm{~F} / 5 \mathrm{M}$ & $4 \mathrm{~F} / 6 \mathrm{M}$ & $1 \mathrm{~F} / 9 \mathrm{M}$ \\
\hline Age (years) & $57.5 \pm 10.8$ & $56.8 \pm 14.0$ & $61.9 \pm 7.6$ & $71.2 \pm 5.1$ \\
\hline Disease duration (years) & - & $2.1 \pm 1.4$ & $12.2 \pm 4.3$ & $11.2 \pm 3.4$ \\
\hline Handedness (L/AMB/R) & $2 / 1 / 9$ & $1 / 1 / 7$ & $2 / 0 / 8$ & $1 / 0 / 9$ \\
\hline Hoehn-Yahr & - & 1 [1-1.5] (off) & $2[2-3]$ (on) & $2[2-3]$ (on) \\
\hline $\begin{array}{l}\text { Predominant clinical side } \\
\qquad(\mathrm{L} / \mathrm{BIL} / \mathrm{R})^{\mathrm{a}}\end{array}$ & - & $3 / 0 / 6$ & $7 / 1 / 2$ & $4 / 1 / 4$ \\
\hline LEDD & - & - & $670 \pm 270$ & $620 \pm 260$ \\
\hline Levodopa & - & - & $360 \pm 165$ & $401 \pm 186$ \\
\hline Dopamine agonists & - & - & $198 \pm 75$ & $212 \pm 97$ \\
\hline Entacapone & - & - & $360 \pm 398$ & $180 \pm 290$ \\
\hline Amantadine & - & - & $70 \pm 95$ & $25 \pm 54$ \\
\hline Selegiline & - & - & $6 \pm 5$ & $1.5 \pm 3$ \\
\hline UPDRS-III & - & $10.3 \pm 4.7$ (off) & $17.7 \pm 6.7$ (on) & $27.8 \pm 19.4($ on $)$ \\
\hline UPDRS-IV $(32,33,34)$ & - & $0 \pm 0$ & $3.0 \pm 1.7$ & $0 \pm 0$ \\
\hline mAIMS (rest) & - & $0 \pm 0$ & $7.6 \pm 10.6$ & $0 \pm 0$ \\
\hline mAIMS (7 seconds) & - & $0 \pm 0$ & $10.6 \pm 7.5$ & $0 \pm 0$ \\
\hline
\end{tabular}

Values are given as mean \pm SD or as median [interquartile range]. Medication dose is expressed as mg/day. The dose of dopamine agonists represents the dose of pergolide, pramipexole, and ropinirole, converted to a single dose according to the LEDD formula. In addition to the medication listed in the table, 1 patient (with LID) took trihexyphenidyl ( $8 \mathrm{mg} /$ day), 1 patient (without LID) rasagiline (1 mg/day), and 1 patient (without LID) tolcapone (300 mg/day). Key: AMB, ambidextrous; BIL, bilateral; F, female; L, left; LEDD, levodopa equivalent daily dose; LID, levodopa-induced dyskinesias; M, male; mAIMS, modified Abnormal Involuntary Movement Scale score at rest and during serial 7 subtraction task (7 seconds); PD, Parkinson's disease; R, right; UPDRS, Unified Parkinson's Disease Rating Scale; UPDRS-III, motor subscale of the UPDRS; UPDRS-IV, score on UPDRS dyskinesia items 32-34.

a As based on UPDRS-III scores.

When the 3 PD groups were considered separately, this cluster remained significant at the same $p$-level for each of the 3 groups compared with controls (Fig. 1C). This reduction of CB1 availability was not correlated with disease duration (SPM correlation analysis with disease duration as covariate), even at a liberal threshold of $p_{\text {height }}<0.01$ [uncorrected]).

No region displayed greater absolute CB1 availability in PD than in controls.

We then performed an analysis where regional $\left[{ }^{18} \mathrm{~F}\right]$ MK-9470 availability was scaled relatively to the global mean per subject. In this relative analysis, PD patients again showed only 1 highly significant cluster of decrease of CB1 availability in the ventral midbrain ( $p_{\text {height }}<0.05$ corrected for multiple comparisons; Supplementary Table 1B). When early PD patients were compared with controls, clusters of significantly increased CB1 availability in PD were observed in the putamen, anterior insula, prefrontal cortex, hippocampal area, and midcingulate $\left(p_{\text {height }}<0.001\right.$; Fig. 2A, Supplementary Table 1C). Interestingly, the putaminal changes were only significant on the side contralateral to the clinically affected body side. Similar clusters were found when comparing the advanced PD group with controls, with additional clusters in the lower prefrontal and midcingulate cortex. Putaminal changes were now bilateral, although still most pronounced contralaterally to the clinically most affected body side (Fig. 2B, Supplementary Table 1D).

\subsection{Relation between cerebral CBI availability and LID}

We found no significant differences in regional $\mathrm{CB} 1$ availability between the advanced PD patients with and without LID. We also searched for correlations between CB1 availability and severity of LID within the group of advanced PD patients with LID. However, there was no significant correlation between $\mathrm{CB} 1$ availability and $\mathrm{mAIMS}$ scores or scores on the dyskinesia items of the UPDRS-IV subscale (items 32-34).

None of the above results were different when performed with age, gender, levodopa dose, and dose of dopamine agonists as nuisance variables.

\section{Discussion}

In this study we have imaged CB1 in PD brains in vivo. Previous studies of CB1 expression levels in PD have relied either on PD animal models, which only imperfectly recapitulate features of human $\mathrm{PD}$, or on postmortem human PD brain tissue, which can be affected by postmortem protein degradation or tissue handling artifacts. An additional problem with postmortem studies of CB1 levels in PD is that it is difficult to determine whether the observed changes are due to the disease itself or to chronic dopaminergic treatment.

We have found significant in vivo changes in CB1 availability in PD patients compared with healthy controls. The CB1 changes showed remarkable regional heterogeneity. This contrasts with Huntington's disease, where we recently 


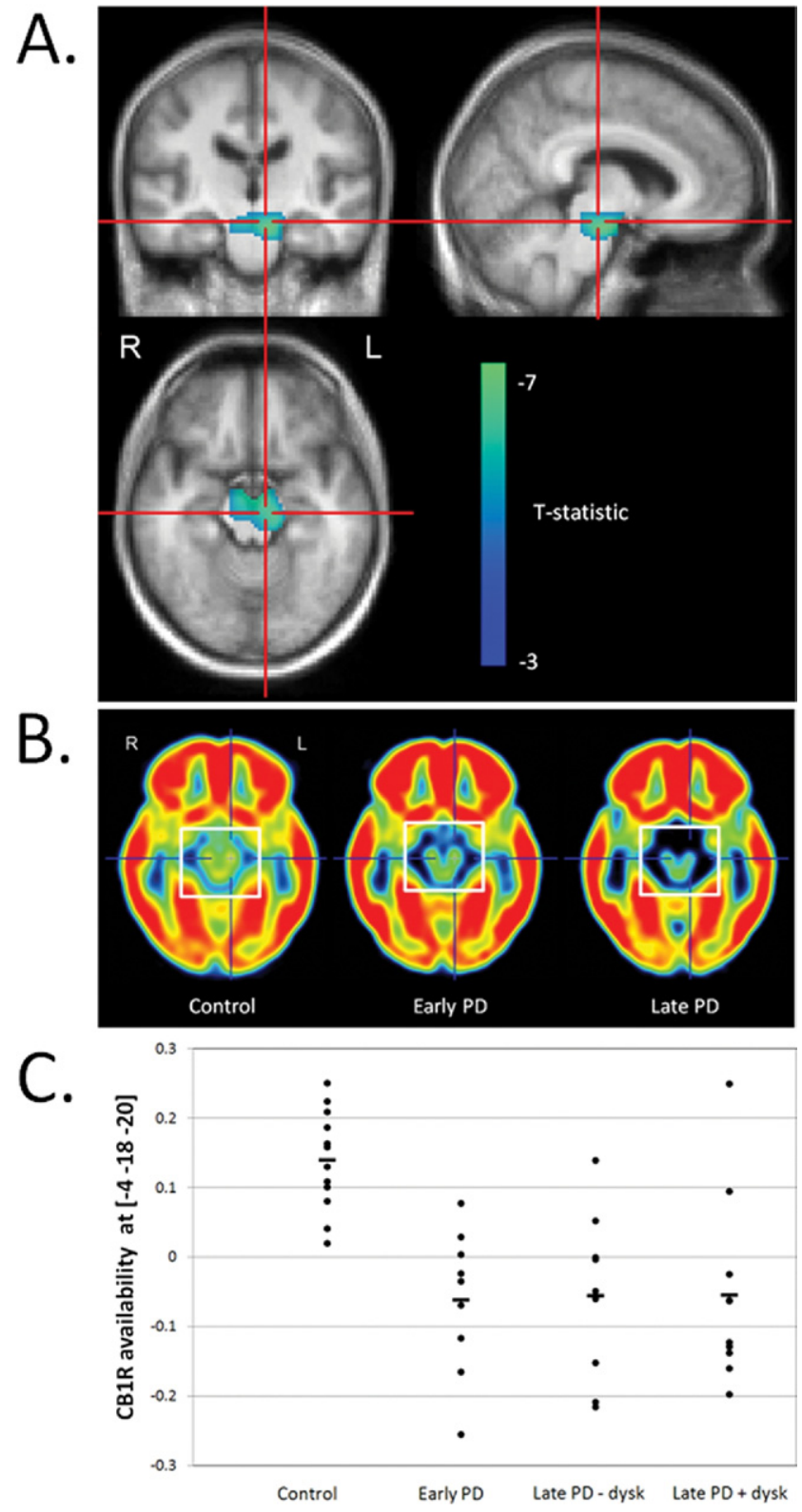

Fig. 1. Decrease in type 1 cannabinoid receptor $(\mathrm{CB} 1)$ availability in the ventral midbrain in Parkinson's disease (PD). (A) Statistical parametric mapping (SPM) analysis of the absolute decrease in CB1 availability in PD patients $\left(p_{\text {height }}<0.05\right.$, corrected) superimposed on an average patient magnetic resonance image (MRI). Image data were flipped so that "left" corresponds to the clinically most affected hemicorpus. (B) Mean transverse images at the level of the ventral mesencephalon for controls (left), early PD (middle) and advanced PD (right), scaled to the same cortical intensity. (C) CB1 availability (relative difference to the mean value for all groups) at the cluster's maximum voxel $([\mathrm{x}, \mathrm{y}, \mathrm{z}]=[4,-18,-20])$.

demonstrated a rather uniform reduction of CB1 availability throughout the entire gray matter (Van Laere et al., 2010).

Compared with healthy controls, PD patients had a profound reduction of $\mathrm{CB} 1$ availability in a ventral mesencephalic region comprising the bilateral substantia nigra. The spatial resolution of PET does not allow to determine the precise anatomical structure or cellular elements responsible for this change. One could speculate that this reduction might result from pruning of target-deprived CB1-expressing presynaptic terminals in the pars compacta of the substantia nigra as a consequence of the death of neuronal targets in this nucleus. However, previous studies have indicated that $\mathrm{CB} 1$ expression in the pars compacta is normally very low, whereas most $\mathrm{CB} 1$ in the substantia nigra is typically found in the pars reticulata (Herkenham et al., 1990, 1991b; Matyas et al., 2006). Therefore, the observed ventral mesencephalic CB1 deficiency probably originates in the pars reticulata rather than the pars compacta. This reduction of CB1 availability occurs early in PD and does not correlate with disease duration.

$\mathrm{CB} 1$ receptors in the pars reticulata have been shown to be present on presynaptic terminals of striatal medium spiny neurons projecting to the pars reticulata (Herkenham et al., 1991a; Mailleux and Vanderhaeghen, 1992; Matyas et al., 2006). Interestingly, several studies have reported reduced $\mathrm{CB} 1$ messenger ribonucleic acid (mRNA) expression in the striatum of $\mathrm{PD}$ animal models and postmortem striatum from PD patients (Hurley et al., 2003; Silverdale et al., 2001). Reduced CB1 mRNA expression in striatal medium

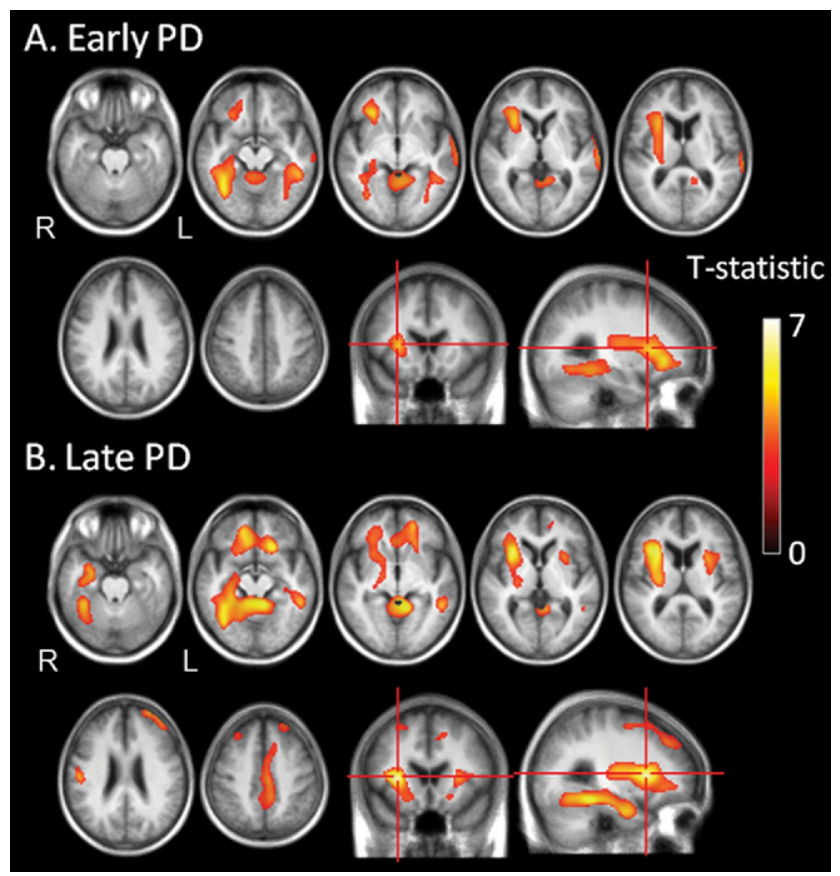

Fig. 2. Increase in type 1 cannabinoid receptor (CB1) availability in dopaminergic projection areas in Parkinson's disease (PD). Image data were flipped so that "left" corresponds to the clinically most affected hemicorpus. (A) Relative increases in CB1 availability in early PD (early PD $>$ controls, at $p_{\text {height }}<0.001$ uncorrected). Significant clusters are observed in the putamen, anterior insula, and hippocampal area. In the putamen, changes were only significant contralaterally to the clinically affected hemicorpus. (B) Relative increases in CB1 availability in advanced PD (late PD $>$ controls, at $p_{\text {height }}<0.001$ uncorrected). Significant clusters are seen bilaterally in the putamen, anterior insula, prefrontal cortex, hippocampal area, and midcingulate. 
spiny neurons would be expected to lead to reduced expression of $\mathrm{CB} 1$ receptors on the axon terminals projecting from these neurons to the pars reticulata and could thus underlie our finding of reduced CB1 availability in the PD substantia nigra.

Previous studies on CB1 protein levels in the substantia nigra of $\mathrm{PD}$ animal models and postmortem substantia nigra from PD patients have provided mixed findings. Walsh et al. (2010) found that intrastriatal injection of 6-hydroxydopamine in rats led to a profound reduction of immunohistochemical CB1 staining in the pars reticulata of the substantia nigra, consistent with our results in PD patients in vivo; however, 6-hydroxydopamine injection into nigrostriatal axons at the level of the medial forebrain bundle had no effect on CB1 levels in the pars reticulata (Walsh et al., 2010). Garcia-Arencibia et al. (2009) measured CB1 protein levels in genetic mouse models of PD and observed a biphasic response in the substantia nigra: $\mathrm{CB} 1$ receptor binding was reduced in mice under 1 year of age, followed by an upregulation of CB1 in older mice. Finally, LastresBecker et al. (2001) found no significant change in CB1 protein levels in postmortem substantia nigra tissue from PD patients, although a trend toward reduction was observed. Importantly, CB1 receptor levels in the last mentioned studies by Garcia-Arencibia et al. (2009) and LastresBecker et al. (2001) were measured using an assay based on binding of the $\mathrm{CB} 1$ agonist $\left[{ }^{3} \mathrm{H}\right] \mathrm{CP}-55,940$. However, $\left[{ }^{3} \mathrm{H}\right] \mathrm{CP}-55,940$ is not selective for $\mathrm{CB} 1$, but binds with similar affinity to $\mathrm{CB} 2$ receptors. $\mathrm{CB} 2$ in brain is mainly expressed on activated microglia (Stella, 2010). Because loss of substantia nigra neurons in PD is associated with microglial activation (Ouchi et al., 2005), CB2 levels may become upregulated in the substantia nigra in PD, which would confound measurements of $\mathrm{CB} 1$ levels based on $\left[{ }^{3} \mathrm{H}\right] \mathrm{CP}-55,940$ binding. By contrast, $\left[{ }^{18} \mathrm{~F}\right] \mathrm{MK}-9470$, the radioligand used in our present study, is highly selective for CB1 (Burns et al., 2007).

In contrast to the reduction in $\mathrm{CB} 1$ availability in the substantia nigra, we found a relative increase of CB1 in the putamen in PD, which is in line with previous postmortem studies of MPTP-treated monkeys and human PD patients (Lastres-Becker et al., 2001). Interestingly, this increase was observed only contralaterally to the motor symptoms in early PD patients with unilateral clinical signs and continued to be most pronounced contralaterally to the clinically most affected hemicorpus in advanced patients with bilateral signs. In the normal striatum CB1 is not expressed on nigrostriatal dopaminergic terminals, but is mainly found on presynaptic glutamatergic terminals originating in the cortex (Benarroch, 2007; Brotchie, 2003; Piomelli, 2003; Uchigashima et al., 2007). These presynaptic CB1 receptors, like $\mathrm{D}_{2}$ dopamine receptors, reduce glutamate release from corticostriatal synapses (Yin and Lovinger, 2006). Upregulation of this $\mathrm{CB} 1$ population in $\mathrm{PD}$ could thus represent a compensatory response, dampening the excessive corticos- triatal glutamatergic drive that results from dopamine depletion (Benarroch, 2007; Brotchie, 2003). Interestingly, cerebrospinal fluid (CSF) levels of the endocannabinoid anandamide were recently found to be increased in untreated PD patients, consistent with a compensatory upregulation of the ECS in PD (Pisani et al., 2010). In addition to its presence on presynaptic corticostriatal terminals, $\mathrm{CB} 1$ in the striatum is also expressed on synapses between medium spiny neurons and between interneurons and medium spiny neurons (Uchigashima et al., 2007), and these CB1 populations could also contribute to the observed changes in striatal CB1 availability.

Unexpectedly, we also observed increased CB1 availability in the anterior insula, prefrontal cortex, hippocampus, and midcingulate in PD patients compared with controls. A common feature of these mesolimbic and mesocortical brain regions is that they receive dopaminergic innervation from the ventral tegmental area (VTA). Dopaminergic neurons in the VTA degenerate in PD, although less so than those in the substantia nigra (Hirsch et al., 1988). Thus, our findings suggest upregulation of CB1 availability in most of the brain regions affected by dopamine depletion in PD. As CB1 is not expressed by VTA dopaminergic neurons (van der Stelt and Di Marzo, 2003), the observed increase of CB1 availability in mesolimbic and mesocortical regions likely reflects an upregulation of CB1 on presynaptic glutamatergic or GABAergic terminals. CB1 plays an important role in the long-loop feedback connections of the mesocorticolimbic pathway (van der Stelt and Di Marzo, 2003). CB1 activation in mesolimbic projection areas disinhibits the VTA dopaminergic cells, increasing their firing rate and triggering dopamine release (Robbe et al., 2001). Therefore, the observed upregulation of CB1 availability in mesolimbic and mesocortical areas could represent a compensatory response to dopamine depletion. Given the involvement of mesocortical and mesolimbic areas in cognition and emotion, the observed CB1 changes in these regions could be related to the cognitive and psychiatric symptoms of PD. Further investigation of regional CB1 availability in PD patients more extensively characterized in terms of cognitive performance, anxiety, mood problems, apathy, psychosis, and impulse control disorders is therefore warranted.

Our second aim was to investigate the relation between CB1 availability and LID, given the previously suggested role for the ECS in the generation of LID (Fox et al., 2002; Sieradzan et al., 2001). A randomized, double-blind, placebo-controlled, crossover trial in 7 PD patients showed that the CB1 agonist nabilone reduced LID (Sieradzan et al., 2001), although a subsequent randomized, double-blind, placebo-controlled, crossover trial of oral cannabis extract in $19 \mathrm{PD}$ patients failed to find an antidyskinetic effect (Carroll et al., 2004). We found no differences in regional CB1 availability between advanced PD patients with and without LID. The 
average age difference between the advanced PD groups with and without LID was 9 years, which might have confounded the results. However, this potentially confounding effect should be minimal, because age-dependence of $\left[{ }^{18} \mathrm{~F}\right]$ MK-9470 availability is negligible after the age of 50 (Van Laere et al., 2008). Furthermore we have formally included age as nuisance variable in the analysis, to exclude such potential confound, but found no differences with and without nuisance variable inclusion. We also found no significant correlations between regional $\mathrm{CB} 1$ availability and LID severity within the group of subjects with LID. A caveat is that LID severity in PD is difficult to quantify, as the mAIMS, UPDRS-IV, and other dyskinesia scales have intrinsic limitations as rating instruments for LID (Fabbrini et al., 2007). Despite these limitations, our data do not support a role for dysregulation of CB1 availability in the development of LID.

The changes in $\left[{ }^{18} \mathrm{~F}\right] \mathrm{MK}-9470$ binding in the ventral midbrain and the dopaminergic projection areas are not caused by the use of dopaminergic drugs, because they were also observed in drug-naïve patients. Also, inclusion of equivalent levodopa dosage and dopamine agonist dosage did not alter the observed results. Furthermore, studies in healthy rats have shown that chronic dopaminergic treatment does not affect binding of $\left[{ }^{18} \mathrm{~F}\right] \mathrm{MK}-9470$ to $\mathrm{CB} 1$ (Casteels et al., 2010). Preclinical data indicate that augmentation of endocannabinoid tone also does not alter $\left[{ }^{18} \mathrm{~F}\right]$ MK-9470 binding (Van Laere, unpublished observations), similar to other $\mathrm{CB} 1$ tracers with same affinity range and binding site such as ${ }^{11} \mathrm{C}-\mathrm{MPePP}$ (Terry et al., 2008). This is likely due to the very high affinity of CB1 for these ligands compared with endocannabinoids. Therefore, changes in $\left[{ }^{18} \mathrm{~F}\right] \mathrm{MK}-9470$ binding primarily reflect changes in $\mathrm{CB} 1$ levels, rather than changes in synaptic concentration of endocannabinoids.

In conclusion, we have found significant regional alterations in $\mathrm{CB} 1$ availability in PD brains in vivo but no relation to LID. Given the observed upregulation of CB1 availability in mesolimbic and mesocortical regions in $\mathrm{PD}$, further exploration of the role of CB1 in the neuropsychiatric problems of PD is warranted.

\section{Disclosure statement}

The authors declare they have no conflict of interest.

All subjects gave written informed consent for the study. The study was conducted in accordance with the Declaration of Helsinki and was also approved by the local Ethics Committee.

\section{Acknowledgements}

The authors thank the study subjects for their willingness to participate. The study was supported by the Fund for
Scientific Research-Flanders (FWO/G.0548.06) and the University of Leuven (OT/05/58). KVL and WV are Senior Clinical Investigators of the FWO. The authors thank the PET radiopharmacy and clinical pharmacology personnel for their skilled support. The tracer precursor for MK-9470 was kindly donated by Merck Inc.

\section{Appendix A. Supplementary data}

Supplementary data associated with this article can be found, in the online version, at doi:10.1016/j.neurobiolaging. 2011.02.009.

\section{References}

Benarroch, E., 2007. Endocannabinoids in basal ganglia circuits: implications for Parkinson disease. Neurology 69, 306-309.

Berrendero, F., Sepe, N., Ramos, J.A., Di Marzo, V., Fernandez-Ruiz, J.J., 1999. Analysis of cannabinoid receptor binding and mRNA expression and endogenous cannabinoid contents in the developing rat brain during late gestation and early postnatal period. Synapse 33, 181-191.

Bisogno, T., Berrendero, F., Ambrosino, G., Cebeira, M., Ramos, J.A., Fernandez-Ruiz, J.J., Di Marzo, V., 1999. Brain regional distribution of endocannabinoids: implications for their biosynthesis and biological function. Biochem. Biophys. Res. Commun. 256, 377-380.

Briggs, G.G., Nebes, R.D., 1975. Patterns of hand preference in a student population. Cortex 11, 230-238.

Brotchie, J.M., 2003. CB1 cannabinoid receptor signalling in Parkinson's disease. Curr. Opin. Pharmacol. 3, 54-61.

Burns, H.D., Van Laere, K., Sanabria-Bohorquez, S., Hamill, T.G., Bormans, G., Eng, W.S., Gibson, R., Ryan, C., Connolly, B., Patel, S., Krause, S., Vanko, A., Van Hecken, A., Dupont, P., De Lepeleire, I., Rothenberg, P., Stoch, S.A., Cote, J., Hagmann, W.K., Jewell, J.P., Lin, L.S., Liu, P., Goulet, M.T., Gottesdiener, K., Wagner, J.A., de Hoon, J., Mortelmans, L., Fong, T.M., Hargreaves, R.J., 2007. [18F]MK-9470, a positron emission tomography (PET) tracer for in vivo human PET brain imaging of the cannabinoid-1 receptor. Proc. Natl. Acad. Sci. U. S. A. 104, 9800-9805.

Carroll, C.B., Bain, P.G., Teare, L., Liu, X., Joint, C., Wroath, C., Parkin, S.G., Fox, P., Wright, D., Hobart, J., Zajicek, J.P., 2004. Cannabis for dyskinesia in Parkinson disease: a randomized double-blind crossover study. Neurology 63, 1245-1250.

Casteels, C., Vanbilloen, B., Vercammen, D., Bosier, B., Lambert, D.M., Bormans, G., Van Laere, K., 2010. Influence of chronic bromocriptine and levodopa administration on cerebral type 1 cannabinoid receptor binding. Synapse 64, 617-623.

Di Marzo, V., Matias, I., 2005. Endocannabinoid control of food intake and energy balance. Nat. Neurosci. 8, 585-589.

Fabbrini, G., Brotchie, J.M., Grandas, F., Nomoto, M., Goetz, C.G., 2007. Levodopa-induced dyskinesias. Mov. Disord. 22, 1379-1389.

Fox, S.H., Brotchie, J.M., Lang, A.E., 2008. Non-dopaminergic treatments in development for Parkinson's disease. Lancet Neurol. 7, 927-938.

Fox, S.H., Henry, B., Hill, M., Crossman, A., Brotchie, J., 2002. Stimulation of cannabinoid receptors reduces levodopa-induced dyskinesia in the MPTP-lesioned nonhuman primate model of Parkinson's disease. Mov. Disord. 17, 1180-1187.

Garcia-Arencibia, M., Garcia, C., Kurz, A., Rodriguez-Navarro, J.A., Gispert-Sachez, S., Mena, M.A., Auburger, G., de Yebenes, J.G., Fernandez-Ruiz, J., 2009. Cannabinoid CB1 receptors are early downregulated followed by a further upregulation in the basal ganglia of mice with deletion of specific park genes. J. Neural Transm. Suppl., 269-275. 
Guy, W., 1976. ECDEU Assessment Manual for Psychopharmacology, US Department of Health, Eduation and Welfare, Rockville, MD.

Haznedar, M.M., Buchsbaum, M.S., Hazlett, E.A., Licalzi, E.M., Cartwright, C., Hollander, E., 2006. Volumetric analysis and three-dimensional glucose metabolic mapping of the striatum and thalamus in patients with autism spectrum disorders. Am. J. Psychiatry 163, 12521263.

Herkenham, M., Lynn, A.B., De Costa, B.R., Richfield, E.K., 1991a. Neuronal localization of cannabinoid receptors in the basal ganglia of the rat. Brain Res. 547, 267-274.

Herkenham, M., Lynn, A.B., Johnson, M.R., Melvin, L.S., De Costa, B.R., Rice, K.C., 1991b. Characterization and localization of cannabinoid receptors in rat brain: a quantitative in vitro autoradiographic study. J. Neurosci. 11, 563-583.

Herkenham, M., Lynn, A.B., Little, M.D., Johnson, M.R., Melvin, L.S., De Costa, B.R., Rice, K.C., 1990. Cannabinoid receptor localization in brain. Proc. Natl. Acad. Sci. U. S. A. 87, 1932-1936.

Hirsch, E., Graybiel, A.M., Agid, Y.A., 1988. Melanized dopaminergic neurons are differentially susceptible to degeneration in Parkinson's disease. Nature 334, 345-348.

Hurley, M.J., Mash, D.C., Jenner, P., 2003. Expression of cannabinoid CB1 receptor mRNA in basal ganglia of normal and parkinsonian human brain. J. Neural Transm. 110, 1279-1288.

Katona, I., Freund, T.F., 2008. Endocannabinoid signaling as a synaptic circuit breaker in neurological disease. Nat. Med. 14, 923-930.

Kreitzer, A.C., Malenka, R.C., 2007. Endocannabinoid-mediated rescue of striatal LTD and motor deficits in Parkinson's disease models. Nature 445, 643-647.

Lastres-Becker, I., Cebeira, M., De Ceballos, M.L., Zeng, B.Y., Jenner, P., Ramos, J.A., Fernandez-Ruiz, J.J., 2001. Increased cannabinoid CB1 receptor binding and activation of GTP-binding proteins in the basal ganglia of patients with Parkinson's syndrome and of MPTP-treated marmosets. Eur. J. Neurosci. 14, 1827-1832.

Mailleux, P., Vanderhaeghen, J.J., 1992. Distribution of neuronal cannabinoid receptor in the adult rat brain: a comparative receptor binding radioautography and in situ hybridization histochemistry. Neuroscience 48, 655-668.

Matyas, F., Yanovsky, Y., Mackie, K., Kelsch, W., Misgeld, U., Freund, T.F., 2006. Subcellular localization of type 1 cannabinoid receptors in the rat basal ganglia. Neuroscience 137, 337-361.

Ouchi, Y., Yoshikawa, E., Sekine, Y., Futatsubashi, M., Kanno, T., Ogusu, T., Torizuka, T., 2005. Microglial activation and dopamine terminal loss in early Parkinson's disease. Ann. Neurol. 57, 168-175.

Piomelli, D., 2003. The molecular logic of endocannabinoid signalling. Nat. Rev. Neurosci. 4, 873-884.

Pisani, V., Moschella, V., Bari, M., Fezza, F., Galati, S., Bernardi, G., Stanzione, P., Pisani, A., Maccarrone, M., 2010. Dynamic changes of anandamide in the cerebrospinal fluid of Parkinson's disease patients. Mov. Disord. 25, 920-924.

Quarantelli, M., Berkouk, K., Prinster, A., Landeau, B., Svarer, C., Balkay, L., Alfano, B., Brunetti, A., Baron, J.C., Salvatore, M., 2004. Integrated software for the analysis of brain PET/SPECT studies with partialvolume-effect correction. J. Nucl. Med. 45, 192-201.

Robbe, D., Alonso, G., Duchamp, F., Bockaert, J., Manzoni, O.J., 2001. Localization and mechanisms of action of cannabinoid receptors at the glutamatergic synapses of the mouse nucleus accumbens. J. Neurosci. $21,109-116$.

Sanabria-Bohorquez, S., Hamill, T., Goffin, K., De Lepeleire, I., Bormans, G., Burns, H.D., Van Laere, K., 2010. Kinetic analysis of the cannabinoid-1 receptor PET tracer [(18)F]MK-9470 in human brain. Eur. J. Nucl. Med. Mol. Imaging 37, 920-933.

Sieradzan, K.A., Fox, S.H., Hill, M., Dick, J.P., Crossman, A.R., Brotchie, J.M., 2001. Cannabinoids reduce levodopa-induced dyskinesia in Parkinson's disease: a pilot study. Neurology 57, 2108-2111.

Silverdale, M.A., McGuire, S., McInnes, A., Crossman, A.R., Brotchie, J.M., 2001. Striatal cannabinoid CB1 receptor mRNA expression is decreased in the reserpine-treated rat model of Parkinson's disease. Exp. Neurol. 169, 400-406.

Stella, N., 2010. Cannabinoid and cannabinoid-like receptors in microglia, astrocytes, and astrocytomas. Glia 58, 1017-1030.

Terry, G., Liow, J.S., Chernet, E., Zoghbi, S.S., Phebus, L., Felder, C.C., Tauscher, J., Schaus, J.M., Pike, V.W., Halldin, C., Innis, R.B., 2008. Positron emission tomography imaging using an inverse agonist radioligand to assess cannabinoid CB1 receptors in rodents. Neuroimage 41, 690-698.

Thie, J.A., Hubner, K.F., Isidoro, F.P., Smith, G.T., 2007. A weight index for the standardized uptake value in 2-deoxy-2-[F-18]fluoro-D-glucose-positron emission tomography. Mol. Imaging Biol. 9, 91-98.

Uchigashima, M., Narushima, M., Fukaya, M., Katona, I., Kano, M., Watanabe, M., 2007. Subcellular arrangement of molecules for 2-arachidonoyl-glycerol-mediated retrograde signaling and its physiological contribution to synaptic modulation in the striatum. J. Neurosci. 27, 3663-3676.

van der Stelt, M., Di Marzo, V., 2003. The endocannabinoid system in the basal ganglia and in the mesolimbic reward system: implications for neurological and psychiatric disorders. Eur. J. Pharmacol. 480, 133150.

Van Laere, K., Casteels, C., Dhollander, I., Goffin, K., Grachev, I., Bormans, G., Vandenberghe, W., 2010. Widespread decrease of type 1 cannabinoid receptor availability in Huntington disease in vivo. J. Nucl. Med. 51, 1413-1417.

Van Laere, K., Goffin, K., Bormans, G., Casteels, C., Mortelmans, L., de Hoon, J., Grachev, I., Vandenbulcke, M., Pieters, G., 2009. Relationship of type 1 cannabinoid receptor availability in the human brain to novelty-seeking temperament. Arch. Gen. Psychiatry 66, 196-204.

Van Laere, K., Goffin, K., Casteels, C., Dupont, P., Mortelmans, L., de Hoon, J., Bormans, G., 2008. Gender-dependent increases with healthy aging of the human cerebral cannabinoid-type 1 receptor binding using [(18)F]MK-9470 PET. Neuroimage 39, 1533-1541.

Walsh, S., Mnich, K., Mackie, K., Gorman, A.M., Finn, D.P., Dowd, E., 2010. Loss of cannabinoid CB1 receptor expression in the 6-hydroxydopamine-induced nigrostriatal terminal lesion model of Parkinson's disease in the rat. Brain Res. Bull. 81, 543-548.

Wilson, R.I., Nicoll, R.A., 2002. Endocannabinoid signaling in the brain. Science 296, 678-682.

Yin, H.H., Lovinger, D.M., 2006. Frequency-specific and D2 receptormediated inhibition of glutamate release by retrograde endocannabinoid signaling. Proc. Natl. Acad. Sci. U. S. A. 103, 8251-8256. 